Hautarzt 2017 · 68:80-82

DOI 10.1007/s00105-016-3889-9

Online publiziert: 11 . November 2016

(c) Der/die Autor(en) 2016. Dieser Artikel ist

eine Open-Access-Publikation.

CrossMark

\author{
V. Ahlgrimm-Siess ${ }^{1} \cdot$ R. Hofmann-Wellenhof ${ }^{2} \cdot$ M. Laimer ${ }^{1}$ \\ ' Universitätsklinik für Dermatologie, Paracelsus Private Medizinische Universität Salzburg, Salzburg, \\ Österreich \\ ${ }^{2}$ Abteilung für Dermatologie und Venerologie, Medizinische Universität Graz, Graz, Österreich
}

\title{
Typisch androgenetische Alopezie?
} für eine androgenetische Alopezie vom weiblichen Typ, aber nicht diagnostisch. Die Trichoskopie (Dermatoskopie von Haar und Kopfhaut) kann wichtige differenzialdiagnostische Hinweise liefern.

\section{Fall 1}

Die 60-jährige Patientin zeigte klinisch eine ausgeprägte ovalförmige Haarlichtung im Bereich des Oberhauptes mit Aussparung der vorderen Haarlinie (• Abb. 1a). Die in diesem Bereich gut sichtbare Kopfhaut wies einen atrophisch glänzenden Aspekt mit diskreten perifollikulären Rötungen um einzelne verbliebene Haarschäfte auf.

Auflichtmikroskopisch waren in diesem Bereich neben dem Verlust von Follikelöffnungen ein Erythem mit perifollikulärer Betonung um Resthaare (- Abb. 2a, weißer Pfeil) und weißliche Areale (• Abb. 2a, schwarzer Pfeil) erkennbar.

\section{Fall 2}

Klinisch präsentierte die 29-jährige $\mathrm{Pa}$ tientin eine ovaläre, mäßig ausgeprägte Haarlichtung im Bereich des Scheitels und der angrenzenden Kopfhaut (- Abb. 1b).

In der dermatoskopischen Untersuchung des betroffenen Areals waren eine deutliche Variabilität der Haarschaftdicke (- Abb. 2b, weiße Pfeile) und das Überwiegen von Follikelostien mit nur einem austretenden Haarschaft (• Abb. 2b, Pfeilspitzen) als Korrelat für die klinisch sichtbare Haarlichtung zu erkennen.

\section{Fall 3}

Selten können andere Haarerkrankungen das Bild einer androgenetischen Alopezie imitieren, allen voran die Alopecia areata und der Lichen planopilaris [2].

\section{Fall 1}

Die 26-jährige Patientin zeigte klinisch eine diffuse Haarlichtung mit stärkster Ausprägung im Scheitelbereich, wodurch hier die Kopfhaut sichtbar war. Diese wies klinisch zahlreiche, durch Dilatation prominent erscheinende Follikelöffnungen mit vereinzelt komedoartigem Aspekt auf.

Dermatoskopisch waren neben der Rarefizierung der Haarschäfte auch kurze, zum Teil nach proximal konisch zulaufende Haare mit stumpfen Enden (• Abb. 2c, „Ausrufezeichenhaare“, schwarze Pfeile) zu erkennen. Zahlreiche Follikelöffnungen sowohl haarloser als auch haartragender Follikel waren dilatiert und mit einem gelblichen Material gefüllt (• Abb. 2c, „yellow dots“, weiße Pfeile).

\section{Diskussion}

Haarausfall kann klinisch diffus, fokal oder in einem sog. Muster vorliegen. „Haarausfall in einem Muster “ findet sich typischerweise bei der androgenetischen Alopezie. Frauen zeigen am häufigsten eine ovaläre oder trianguläre Ausdünnung des Scheitelbereichs bei erhaltener frontaler Haarlinie (Typ Ludwig und Olsen), ein männliches Ausfallsmuster wird in Einzelfällen beobachtet (Typ Hamilton) [1].
Bei unserer ersten Patientin (Fall 1; - Abb. 1a und 2a) zeigte sich ein mit einer fortgeschrittenen androgenetischen Alopezie vom Typ Ludwig vereinbares Haarausfallsmuster, die Veränderungen der Kopfhaut waren jedoch bereits klinisch suspekt für einen vernarbenden Prozess. Der Verlust von Follikelöffnungen und das Vorliegen weißlich fibrosierter Areale in der Auflichtmikroskopie erhärteten diesen Verdacht. Das perifollikulär betonte Erythem im Bereich von Resthaaren war hinweisend für einen Lichen planopilaris (•Tab. 1). Die Verdachtsdiagnose bestätigte sich auch histologisch.

Die sog. „fibrosing alopecia in a pattern distribution" stellt eine klinische Variante des Lichen planopilaris dar, die in für die androgenetische Alopezie typischen Arealen auftritt.

Die dermatoskopische Untersuchung der Kopfhaut ist für die Unterscheidung vernarbender und nicht vernarbender Alopezien von großer Bedeutung. Der gleichzeitige Verlust von Haaren und Follikelöffnungen stellt das Hauptmerkmal der primär vernarbenden Alopezien dar. Weitere auflichtmikroskopische Befunde helfen bei der Unterscheidung der Subentitäten; die perifollikuläre Betonung von Erythem, Fibrosierung und Schuppung mit zum Teil halskrausenartigem Aspekt ist typisch für den Lichen 

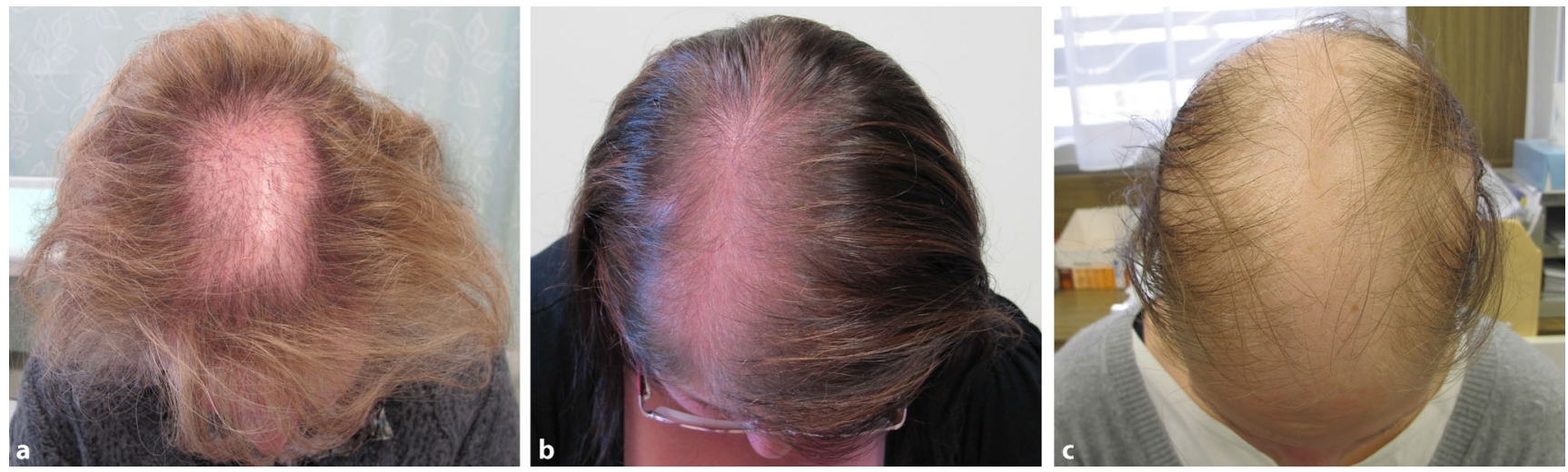

Abb. 1a-c $\Delta$ Drei Patientinnen mit am Oberhaupt betonter Haarlichtung wie bei androgenetischer Alopezie vom weiblichen Typ
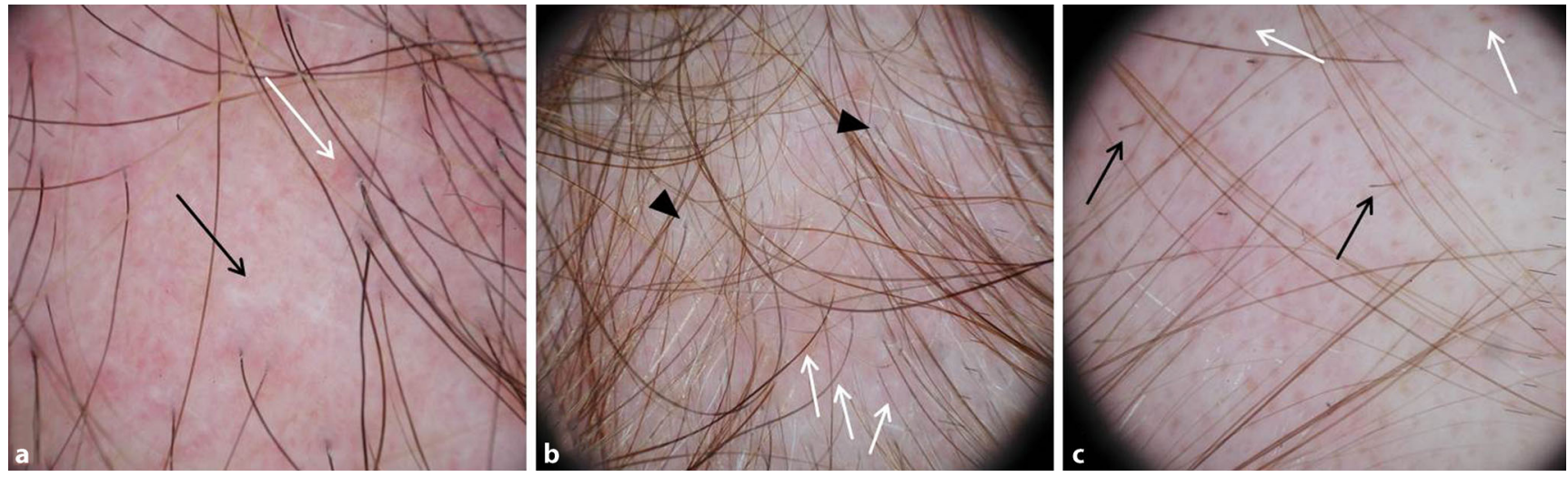

Abb. 2 A Dermatoskopische Aufnahmen der in Abb. 1 gezeigten Patientinnen. a Verlust von Follikelöffnungen mit Vorliegen weißlich fibrosierter Areale (schwarzer Pfeil) und charakteristisches perifollikuläres Erythem (weißer Pfeil). b Variabilität der Haarschaftdicke durch Vorliegen normaler Terminalhaare, intermediärer und dünner Haare (weiße Pfeile) sowie Zunahme von Vellushaaren und von Follikelostien mit Austritt von Einzelhaaren (Pfeilspitzen). c Kurze, sich zum Teil zur Kopfhaut hin verjüngende Haare mit stumpfen Enden (schwarze Pfeile , „Ausrufezeichenhaare“) und deutlich erkennbare, mit gelblichem Material gefüllte Follikelostien (weißePfeile, "yellow dots")

planopilaris [3]. Wegen des gemeinsam zugrunde liegenden entzündlich fibrosierenden Prozesses können vernarbende Alopezien jedoch überlappende auflichtmikroskopische Kriterien zeigen.

\section{》) Diagnose Fall 1: Lichen planopilaris („fibrosing alopecia in a pattern distribution")}

In Hinblick auf therapeutische Konsequenzen empfiehlt sich eine bioptische Diagnosesicherung.

\section{Fall 2}

Bei unserer zweiten Patientin (Fall 2; - Abb. 1 b und 2b) lag klinisch eine Haarlichtung wie bei beginnender androgenetischer Alopezie vom Typ Ludwig vor.
Die auflichtmikroskopisch erfassbare Zunahme von dünnen Haaren und die Abnahme von Follikelostien mit 2 oder 3 gemeinsam austretenden Haarschäften spiegelten eine progressive Miniaturisierung der Haarfollikel in diesem Bereich wider und bestätigten die klinische Verdachtsdiagnose einer androgenetischen Alopezie (•Tab. 1).

Die Trichoskopie ermöglicht durch die morphologische Beurteilung von Haaren und Kopfhaut differenzierte Rückschlüsse auf die Pathogenese von Haarausfallserkrankungen und erleichtert damit differenzialdiagnostische Überlegungen.

Haarveränderungen bei der androgenetischen Alopezie kommen durch die genetisch unterschiedlich stark ausgeprägte Empfindlichkeit einzelner Follikel gegenüber Androgenen zustande
[4]. Unter Hormoneinfluss kommt es zur Schrumpfung oder sogar zum Verlust der betroffenen Haarfollikel. In Abhängigkeit von Hormonstatus und Verstoffwechselung von Geschlechtshormonen in Haaren und Haut sind unterschiedliche Kopfhautareale betroffen. Die Okzipitalregion zeigt meist keine oder nur geringe Veränderungen.

\section{I) Diagnose Fall 2: Andro- genetische Alopezie (Typ Ludwig)}

Die topografisch unterschiedlich starke Ausprägung dermatoskopischer Veränderungen ist von zentraler diagnostischer Bedeutung und ermöglicht das Erkennen von Frühformen ohne klinisch ausgeprägtes Ausfallsmuster. 
Tab. 1 Charakteristische auflichtmikroskopische Befunde der gezeigten Patientinnen

\begin{tabular}{|c|c|c|}
\hline $\begin{array}{l}\text { Fall 1: Lichen } \\
\text { planopilaris }\end{array}$ & Fall 2: Androgenetische Alopezie & $\begin{array}{l}\text { Fall 3: Alopecia areata } \\
\text { incognita }\end{array}$ \\
\hline $\begin{array}{l}\text { Verlust von Follikelöffnun- } \\
\text { gen }\end{array}$ & $\begin{array}{l}\text { Variabilität der Haarschaftdi- } \\
\text { cke (Zunahme von dünnen und } \\
\text { Vellushaaren) }\end{array}$ & $\begin{array}{l}\text { Dystrophe Haarschäfte: kur- } \\
\text { ze, zum Teil nach proximal } \\
\text { konisch zulaufende Haare } \\
\text { mit stumpfen Enden }\end{array}$ \\
\hline $\begin{array}{l}\text { Perifollikulär betontes } \\
\text { Erythem }\end{array}$ & $\begin{array}{l}\text { Zunahme von Follikelostien mit nur } \\
\text { einem austretenden Haarschaft }\end{array}$ & Zahlreiche „yellow dots" \\
\hline \multicolumn{3}{|l|}{ Weißliche Areale } \\
\hline aln androgenabhängigen $\mathrm{Ar}$ & & \\
\hline
\end{tabular}

Die Untersuchung mittels Handdermatoskop stellt eine dem Trichogramm zumindest gleichwertige Methode zur Diagnose der androgenetischen Alopezie dar und ist zudem zeitsparend und schmerzlos. Die meisten Videodermatoskope ermöglichen mittels spezieller Computerprogramme die Durchführung eines digitalen Trichogramms, das zusätzlich zur Bestimmung der Anagen/ Telogen-Ratio auch eine Messung des Vellus-/Terminalhaar-Verhältnisses sowie der Haardichte und -dicke erlaubt. Die Möglichkeit zur digitalen Speicherung ist für die Verlaufskontrolle und objektive Evaluierung des Therapieerfolges von großem Vorteil.

\section{Fall 3}

Die Patientin zeigte klinisch eine für die androgenetische Alopezie vom weiblichen Typ charakteristische Betonung der Scheitelregion. Die starke Ausprägung des Haarausfalls war v. a. im Hinblick auf das Alter der Patientin ungewöhnlich, ebenso die dilatierten Follikelöffnungen im stark betroffenen Areal.

Das Vorliegen zahlreicher „yellow dots" sowie charakteristischer dystropher Haarschäfte in der Auflichtmikroskopie führte uns zur Diagnose einer Alopecia areata incognita (• Tab. 1).

\section{) Diagnose Fall 3: Alopecia areata incognita (Alopecia areata diffusa)}

Klinisch zeigt die Alopecia areata incognita häufig ein der androgenetischen Alopezie ähnliches Haarausfallsmuster.

Bei entzündlich bedingtem Haarausfall kann die Krankheitsaktivität häufig auflichtmikroskopisch bestimmt werden. Die Alopecia areata zeigt durch die entzündliche Infiltration der Bulbusregion in aktiven Stadien eine Störung des Haarwachstums; es finden sich typischerweise abgebrochene Haare, sich zur Kopfhaut hin verjüngende Haare und sog. auf Kopfhautniveau kadaverisierte Haare („black dots“) [5]. Die Ansammlung nicht pigmentierter Haarreste und von Sebum führt zur Dilatation und gelblichen Färbung von Follikelöffnungen. Das Auftreten kurzer, nachwachsender Haare ist als prognostisch günstiges Zeichen zu sehen.

\section{Fazit für die Praxis}

- Die Trichoskopie ist in der nichtinvasiven Diagnostik von Haarausfallserkrankungen von unschätzbarem Wert.

- Daneben ermöglicht die Trichoskopie die Diagnose weiterer entzündlicher Erkrankungen der Kopfhaut sowie von (genetischen) Haarschaftanomalien und Ungezieferbefall.

\section{Korrespondenzadresse}

\section{PD. Dr. V. Ahlgrimm-Siess}

Universitätsklinik für Dermatologie, Paracelsus Private Medizinische Universität Salzburg Müllner Hauptstr. 48, 5020 Salzburg, Österreich v.ahlgrimm-siess@salk.at

Open access funding provided by Paracelsus Medical University.

\section{Einhaltung ethischer Richtlinien}

Interessenkonflikt. V. Ahlgrimm-Siess, R. HofmannWellenhof und M. Laimer geben an, dass kein Interessenkonflikt besteht.
Dieser Beitrag beinhaltet keine von den Autoren durchgeführten Studien an Menschen oder Tieren. Alle Patienten, die über Bildmaterial oder anderweitige Angaben innerhalb des Manuskripts zu identifizieren sind, haben hierzu ihre schriftliche Einwilligung gegeben.

Open Access. Dieser Artikel wird unter der Creative Commons Namensnennung 4.0 International Lizenz (http://creativecommons.org/licenses/by/4.0/deed. de) veröffentlicht, welche die Nutzung, Vervielfältigung, Bearbeitung, Verbreitung und Wiedergabe in jeglichem Medium und Format erlaubt, sofern Sie den/die ursprünglichen Autor(en) und die Quelle ordnungsgemäß nennen, einen Linkzur Creative Commons Lizenz beifügen und angeben, ob Änderungen vorgenommen wurden.

\section{Literatur}

1. HerskovitzI, Tosti A (2013) Female pattern hair loss. Int JEndocrinol Metab 11(4):e9860

2. Rossi A, lorio A, Di Nunno D, Priolo L, Fortuna MC, Garelli V, Carlesimo M, Calvieri S, Mari E (2015) Conditionssimulating androgenetic alopecia.J Eur Acad Dermatol Venereol 29(7):1258-1264

3. Assouly P, Reygagne P (2009) Lichen planopilaris: update on diagnosis and treatment. Semin Cutan Med Surg 28(1):3-10

4. Rakowska A, Slowinska M, Kowalska-Oledzka E, Olszewska M, Rudnicka L (2009) Dermoscopy in female androgenic alopecia: method standardization and diagnostic criteria. Int J Trichology 1(2):123-130

5. Tosti A, Whiting D, lorizzo M, Pazzaglia M, Misciali C, Vincenzi C, Micali G (2008) The role of scalp dermoscopy in the diagnosis of alopecia areata incognita. JAm Acad Dermatol 59(1):64-67 